\title{
A Functional Variant of CXCL16 Is Associated with Predisposition to Sepsis and MODS in Trauma Patients
}

\section{Jianhui Sun}

Army Medical University

Huacai Zhang

Army Medical University

Di Liu

Army Medical University

Li Cui

Army Medical University

Qiang Wang

Guizhou Medical University

Lebin Gan

Guizhou Medical University

Dalin Wen

Army Medical University

Jun Wang

Guizhou Medical University

Juan Du

Army Medical University

Hong Huang

Army Medical University

Anqiang Zhang

Army Medical University

Jin Deng

Guizhou Medical University

Jianxin Jiang

Army Medical University

Ling Zeng ( $\nabla$ zengling_1025@126.com )

Army Medical University https://orcid.org/0000-0002-9640-9083

Research 
Keywords: Sepsis, CXCL16, Single nucleotide polymorphisms, Trauma, Multiple organ dysfunction Posted Date: October 28th, 2020

DOI: https://doi.org/10.21203/rs.3.rs-71609/v2

License: (c) (1) This work is licensed under a Creative Commons Attribution 4.0 International License. Read Full License 


\section{Abstract}

Background: CXC chemokine famliy genes play an important role in inflammatory and immune diseases. CXCL16 affects the occurrence and development of inflammation through leukocyte chemotaxis, leukocyte adhesion and endotoxin clearance. In this study, we selected a set of tag SNPs in the CXCL16 gene and investigated their clinical relevance to the development of sepsis and multiple organ dysfunction syndrome (MODS) in patients with major trauma in three independent Chinese Han populations.

Methods: A total of 1,620 major trauma patients were enrolled in this study. Among these patients, 920 came from Chongqing in western China, 350 came from Zhejiang Province in eastern China, and 350 came from Guizhou Province in southern China. The improved multiplex ligation detection reaction (iMLDR) method was employed in the genotyping and genetic association analyses to determine the associations between CXCL16 haplotypes and sepsis morbidity rate and higher MOD scores in three cohorts.

Results: The CXCL16 T123V181 haplotype was determined to be associated with increased risk for sepsis morbidity and higher MOD scores in the three cohorts. In vitro chemotactic experiments demonstrated that the T123V181 protein enhanced the chemotaxis of immunocytes. The adhesion ability of THP-1 cells expressing T123V181 to immunocytes was also stronger than those of cells with the other three haplotypes. T123 and V181 altered the structure of the CXCL16 active center, which resulted in changes in protein function and changes in the adhesion and chemotaxis of CXCL16-expressing immunocytes. These structural changes might be responsible for the increased incidence of sepsis and the higher MODS scores.

Conclusions: We demonstrate that genetic variations in the CXCL16 gene regulate the sepsis morbidity rate, and we explore a paradigm for the prewarning diagnosis of sepsis tailored to the genetic information of individual patients. Trial registration: ClinicalTrials.gov, NCT01713205. Registered 18 October 2012, retrospectively registered.

\section{Introduction}

With the continuous improvement of emergency and trauma care technology, the mortality of severe trauma patients caused by trauma itself has been significantly reduced. However, trauma patients often die of posttraumatic complications, including sepsis and MODS (1). Sepsis represents a considerable threat to human health, affects the quality of human life and has a large financial cost. Approximately 14,000 people die of sepsis and MODS every day worldwide, and this number is increasing at a rate of 1.5 - $8.0 \%$ every year. According to an epidemiological survey, the mortality of sepsis has exceeded that of myocardial infarction, which has become the leading cause of death in intensive care units (2-3). In recent years, the mortality of sepsis has remained as high as $30-70 \%$, despite the considerable advances made in anti-infection treatment and organ function support technology (4). Sepsis is a multifactorial 
disease that is regulated by pathogenic microorganisms and genetic factors. Significant differences in susceptibility and prognosis to sepsis were observed among individuals. Sorensen et al. found that genetic factors played a more important role in the prognosis of infectious diseases than in common diseases, such as cancer and cardiovascular diseases. Individuals whose parents died of infectious diseases are 5.8 times more likely to die after infection (5). There were significant differences in cytokine production, bacterial translocation, prognosis and mortality among mice with different gene backgrounds (6). Stuber et al. first reported that polymorphisms of the TNF gene are significantly related to the susceptibility and prognosis of sepsis and organ dysfunction (7). Since that study was reported, extensive research has identified that genetic factors play important roles in the pathogenesis of complications after trauma. Our research group has taken the lead in conducting association studies and functional studies between genomic polymorphisms and the risk of major trauma complications in China. We sequenced the genomes of $27 \mathrm{Han}$ individuals and obtained information on their single nucleotide polymorphisms (SNPs) in sepsis-related genes. Next, through association studies, we identified a series of SNPs that are closely related to the prognosis of sepsis and MODS. These SNPs are primarily located on pattern recognition receptor genes (8-9) and cytokine genes (10-12), which are closely related to the pathophysiological process of trauma complications.

A large number of neutrophils are produced in bone marrow during infection, and they exert an antiinfection effect by reaching the infected site through the circulatory system (13-14). CXC gene family members are the key component of the host defense response, serving to guide neutrophil migration to bacterial infection sites (15). Therefore, CXC chemokine family genes play important roles in inflammatory and immune-related diseases. However, how CXC family members regulate granulogenesis, neutrophil recruitment, and neutrophil mobilization in response to sepsis caused by infection has not been elucidated (16-17). During sepsis, alveolar macrophages are implicated in polymorphonuclear leukocyte recruitment to the lungs. Wang et al. elucidated that the chemokine $\mathrm{C}-\mathrm{X}-\mathrm{C}$ in septic plasma is responsible for the activation of alveolar macrophages (18). Chemokine C-X-C motif ligand 16 (CXCL16) belongs to the CXC family. CXCL16 is expressed in soluble or transmembrane forms and can be observed in many cell types, including inflammatory cells (such as macrophages, neutrophils, dendritic cells and monocytes) and non-inflammatory cells (such as lung epithelial cells and renal cells). CXCL16 has the characteristics of both CC family and CX3C family chemokines. CXCL16 is primarily expressed on the surface of antigen-presenting cells (APCs) and consists of a chemokine domain ( 89 amino acids), a mucin-type stalk ( 110 amino acids), a single-pass transmembrane domain ( $\sim 20$ amino acids), and a cytoplasmic tail ( 27 amino acids) (19-20). CXCL16 is the only ligand of the CXCR6 receptor. Soluble CXCL16 induces the migration of CXCR6+ cells (including Th1 cells, NK cells and activated CD8+ T-cells) (21-22), M2-macrophage infiltration (23), interactions between APC and CD8+ T-cells (24), the cellular immune response and inflammatory response (25), and the development of thymocytes (26). Membranebound CXCL16 can promote the adhesion of CXCR6+ cells (27-28). CXCL16 plays important roles both in the natural immune barrier and in the occurrence and development of autoimmune diseases. Fahy et al. (29) established a model of salmonellae-induced small enteritis in mice to study the mechanism of the CXCL16 immune response in vivo. These researchers showed that CXCL16 induced the expression of 
interferon through the primary immune response to bacterial infection, thereby playing an important role in small enteritis infection regulation. A study showed that CXCL16 on dendritic cells and macrophages regulates bacterial phagocytosis and adhesion of Staphylococcus aureus and E. coli. CXCL16 mediates bacterial recognition, which suggests that CXCL16 is an important chemokine in infectious diseases (30).

Xu et al. observed that the CXCL16 haplotype rs2304973T-rs1050998C-rs3744700G-rs8123A significantly elevated myocardial infarction risk (31). A clinical study indicated that the CXCL16 missense allele haplotype T123V181 was significantly associated with carotid plaque, which may be caused by the impact of CXCL16 protein sequence variation on the interactions between CXCL16 and CXCR6 (32).

We selected a set of tag SNPs within the entire CXCL16 gene and investigated their clinical relevance in relation to the development of sepsis and MODS in patients with major trauma in three independent Chinese Han populations. In this report, we present experimental evidence for the impact of two missense tag SNPs of CXCL16 on sepsis and MODS. Furthermore, we investigated the mechanism by which the CXCL16 genotype T123V181 promotes neutrophil chemotaxis and adhesion. Our findings may help to establish a new strategy for the early warning and diagnosis of severe trauma complications.

\section{Materials And Methods}

\section{Research populations}

A total of 1,620 major trauma patients were enrolled in this study, all of whom were Han Chinese people from Chongqing in western China $(n=920)$, Zhejiang Province in eastern China $(n=350)$ and Guizhou Province in southern China $(n=350)$. Patients were admitted to Daping Hospital of Army Medical University, Chongqing Emergency Medical Center, the Second Affiliated Hospital of Zhejiang University and the Affiliated Hospital of Guizhou Medical University between 2005 and 2019. The inclusion criteria were as follows: 1) age between 18 and 65,2 ) injury severity score (ISS) greater than 16 , and 3 ) survival for more than two days after injury. The exclusion criteria were as follows: 1) combined with penetrating injuries and 2) severe brain injury or preexisting cardiovascular, respiratory, renal, hepatic, hematological or immunological diseases. Ethics approval for this study was obtained from the Ethics and Protocol Review Committees of Army Medical University, Chongqing Emergency Medical Center, Zhejiang University and Guizhou Medical University (Trial registration: ClinicalTrials.gov, NCT01713205. Registered on 18th October 2012, retrospectively registered). Before enrollment, written informed consent was obtained from the patients or their next of kin, which covered the collection of relevant clinical data and explicit DNA analysis. Patient confidentiality was preserved according to the guidelines of the Declaration of Helsinki.

\section{Sepsis and MODS evaluation}

Sepsis is defined as an acute change in total sequential (sepsis-related) organ failure assessment (SOFA) score greater than or equal to 2 points consequent to an infection (33). MOD scores are evaluated on each hospitalization day. Briefly, pulmonary, renal, hepatic, neurological, cardiac and hematological 
parameters were scored from 0 to 4 every day. The MOD scores ranged from 0 to 4 , and the total score ranged from 0 to 24 (six organs). Failure of organ function was considered to be 3 or more points for more than two consecutive days (34). The presence of sepsis and multiple organ dysfunction scores were determined by individuals who did not know the patients' genotypes.

\section{Genotyping}

Genomic DNA was isolated from peripheral whole blood by QuickGene-610L (Fujifilm, Tokyo, Japan). The concentration and purity of DNA samples were checked by a Thermo Scientific Nanodrop ND-1000 spectrophotometer (Isogen Life Science, De Meern, Netherlands). DNA samples were stored at $-80^{\circ} \mathrm{C}$. SNP genotyping was performed by an improved multiplex ligation detection reaction (iMLDR) technique, as described in our previous report (35). Approximately $10 \%$ of the samples were genotyped in duplicate to assess the accuracy of iMLDR. Genotyping was performed by researchers who did not know the patients' clinical data.

\section{Expression plasmid construction}

A plasmid containing human CXCL16-I123A181 CDNA with a $\mathrm{C}$ terminal flag and His-tag was purchased from Vigene Biosciences (Jinan, Shandong Province, China). Plasmids encoding CXCL16-1123V181, T123A181 and T123V181 cDNA mutations were generated by site-directed mutagenesis (Stratagene, La Jolla, CA). The primers for 123T were 5'-TGAGGCCTGAGAAgTTGGGGGGCTGGTAGGAA-3' (forward) and 5'-CAACTTCTCAGGCCTCAGAGGGGGCA-3' (reverse). The primers for $181 \mathrm{~V}$ were 5'-

CCCAaCTGCCAGACTGTGGCCCGCA-3' (forward) and 5'-ACAGTCTGGCAGtTGGGCCTGAGGCTGGGGA -3' (reverse). The sequences of the four plasmids were checked by direct sequencing (BGI Genomics, Beijing, China). To obtain recombinant human CXCL16 proteins, $\mathrm{CHO}$ cells were cultured in a 6-well plate (F12 medium with $10 \% \mathrm{FCS}$, penicillin-streptomycin, $3 \mathrm{mM}$ glutamine). After washing was performed, $\mathrm{CHO}$ cells were cultured in serum-free medium and transfected with $4 \mu \mathrm{g}$ of CXCL16-I123A181, I123V181, T123A181 or T123V181 plasmids. Transfected cells were cultured in serum-free medium for 48 hours. According to the manufacturer's instructions, the supernatants were subsequently purified by a His-bind purification kit (Merck-Millipore, Massachusetts, the United States) to obtain purified CXCL16-I123A181, 1123V181, T123A181 and T123V181 proteins.

\section{Cell chemotaxis and adhesion assay}

The polycarbonate membrane in the middle of the Transwell insert (Corning, New York, the United States) divides the chamber into two parts: the upper chamber and the lower chamber. THP-1 cells (human monocyte cell strain) were cultured in the upper chamber of the Transwell insert. The purified human CXCL16-I123A181, I123V181, T123A181 and T123V181 proteins were dissolved in the medium of the lower chamber $(100 \mathrm{ng} / \mathrm{ml})$. Chemotactic cells that passed through the membrane pores were dyed and subsequently counted to calculate the chemotaxis ability of four kinds of human CXCL16 protein (Fig. 2B). 
For the cell adhesion test, THP-1 cells were cultured in serum-free medium and transfected with $4 \mu \mathrm{g}$ of CXCL16-GFP-I123A181, I123V181, T123A181 and T123V181 expression plasmids. Raw264.7 cells that express CXCL16 receptor- CXC chemokine receptor 6 (CXCR6) were seeded at a density of $1 \times 10^{4}$ in a 6well plate (36). Raw264.7 cells were cultured for 48 hours until they reached $70 \%-80 \%$ confluency and were subsequently cocultured with THP-1 cells expressing CXCL16-GFP-I123A181, I123V181, T123A181 and T123V181. The plate was incubated for 60 minutes at $37^{\circ} \mathrm{C}$ to enable cell binding. Nonadherent THP1 cells were washed away. The plate was read at $488 \mathrm{~nm}$ in a fluorescent plate reader. All assays were performed in triplicate. The number of THP-1 cells that adhered to Raw264.7 cells is presented as the mean fluorescence intensity (MFI).

\section{Molecular modeling of CXCL16 polymorphisms}

Using MAFFT (v7.38) (https://mafft.cbrc.jp/alignment/software/algorithms/algorithms.html), the CXCL16 proteins of 11 species were sequenced, and the evolutionary tree was calculated. The structurally conserved regions and loop regions of CXCL16 were analyzed by SMART (https://www.megasoftware.net/). The optimized structures were compared by PyMOL (version 0.97). Polymorphism Phenotyping v2 (Polyphen2, http://genetics.bwh.harvard.edu/pph2/) was employed to predict the effect of the two mutations. Using Modeler, Phyre2 and Raprotx, the full-length CXCL16 sequence was modeled. Using the wild-type CXCL16 structure as the template, the structure models of I123T and A181V are established. Using GROMACS 5.12, the structural models of I123T and A181V were modeled. The distances of amino acids and the area and volume of the hydrophobic pocket were analyzed using the distance geometry method.

\section{Statistical Analysis}

The relevance of CXCL16 tag SNPs for clinical outcomes was analyzed by three genetic models, that is, the dominant, recessive, and allele-dose models. To assess sepsis risk, adjusting for age, sex, and injury severity for confounding effects, odds ratios with $95 \%$ confidence intervals were calculated by multivariable logistic regression models. Adjusted by age, sex, and injury severity for confounding effects, the association of tag SNPs with MOD scores was determined by linear regression analysis. A P-value < 0.05 after Bonferroni correction for multiple testing was employed to indicate significance. SPSS 13.0 software (SPSS Inc., the United States) was utilized for the statistical analysis. Chemotaxis and adhesion data were compared using One-way analysis of variance (One-way ANOVA).

\section{Results}

\section{CXCL16 I123T and A181V are two tag SNPs that are in strong linkage disequilibrium}

CXCL16 is located on chromosome 17 (Fig. 1A.). In the Chinese Han cohort, 20 SNPs were found in CXCL16. Among these SNPs, 12 had minor allele frequencies (MAFs) greater than or equal to $5 \%$. Twelve SNPs constructed one haplotype block. Based on the analysis of the tagging threshold of $r^{2}$, rs 1050998 (1123T) and rs2277680 (A181V) were selected as tag SNPs. Also, I123T and A181V are located in exon 4, 
which encodes the mucin-like stalk domain of CXCL16. According to the genotyping data of $45 \mathrm{Han}$ Chinese from the HapMap database, the two missense mutations are in strong linkage disquilibrium ( $D^{\prime}=$ $\left.1 ; r^{2}=0.99\right)$ (Fig. 1B.). Furthermore, I123T and A181V were genotyped in the three cohorts' blood samples using the iMLDR method (37).

Major trauma patients from Chongqing $(n=920)$, Zhejiang $(n=350)$ and Guiyang $(n=350)$ were recruited for this study. Both $1123 \mathrm{~T}$ and A181V were determined to be in Hardy-Weinberg equilibrium (HWE) among the three cohorts. Four haplotypes and their frequencies of the three cohorts are shown in Table 2. Notably, I123A181 and T123V181 are the two most common haplotypes out of four possible, and they are in strong linkage equilibrium $\left(D^{\prime}=1 ; r^{2}=0.99\right.$ in both cohorts). This result means that there is almost no recombination between the two SNPs and no recombination in the haplotype region (38).

\section{T123V181 haplotype is associated with an increased risk of sepsis and MODS in major trauma patients}

As 1123T and A181V were determined to be located in one haplotype block and observed to be in strong linkage equilibrium, we further compared their haplotype frequencies according to sepsis morbidity rate and MOD scores among major trauma patients from Chongqing, Zhejiang and Guizhou cohorts. There were no significant differences in gender, age or injury severity score among patients in the three cohorts. Among the four haplotypes, T123V181 was found to be associated with an increased risk for sepsis morbidity in both cohorts $(\mathrm{OR}=1.89,95 \% \mathrm{Cl}=1.82-2.56$, and $P=0.001$ for the Chongqing cohort; $\mathrm{OR}=$ $1.76,95 \% \mathrm{Cl}=1.53-2.18$, and $P=0.004$ for the Zhejiang cohort; $\mathrm{OR}=1.55,95 \% \mathrm{Cl}=1.42-1.96$ and $P=$ 0.012 for the Guizhou cohort, Table 3). Notably, T123V181 carriers also were observed to have significantly higher MOD scores than I123A181 carriers among major trauma patients in the three cohorts ( $P=0.0016$ for the Chongqing cohort; $P=0.002$ for the Zhejiang cohort; $P=0.001$ for the Guizhou cohort, Table 3).

\section{T123V181 haplotype enhances the chemotaxis and adhesion of human monocytes}

Neutrophils, monocytes and macrophages in peripheral blood adhere to endothelial cells during sepsis and then pass through endothelial cells and the matrix layer to the infected site. The migration of inflammatory cells during sepsis facilitates the elimination of bacteria, but it also leads to organ dysfunction. Organ dysfunction caused by sepsis is primarily caused by organ damage caused by excessive inflammatory cell recruitment to noninflammatory sites. The two missense mutations rs 1050998 (1123T) and rs2277680 (A181V) confer T $\rightarrow C$ and $G \rightarrow$ A substitutions at positions 4585486 and 4585312 on chromosome 17, leading to a substitution of isoleucine to threonine at codon 123 (1123T) and alanine to valine at codon 181 (A181V) at opposing ends of the mucin-type stalk region (39). Thus, we further elucidated the effect of four haplotypes of the two missense mutations on the chemotaxis and adhesion of human monocytes. Expression plasmids encoding human CXCL16I123A181, I123V181, T123A181 and T123V181 cDNA were transfected into CHO cells to obtain purified soluble CXCL16 proteins (Fig. 2A and 2B). The results of this experiment showed that significantly more THP-1 cells migrated to the low chamber when the chemotactic protein in the low chamber was CXCL16- 
T123V181 ( $\mathrm{P}$ < 0.01) (Fig. 2C). To verify the cell adhesion ability caused by the missense mutation, THP-1 cells transfected with CXCL16-GFP-I123A181, I123V181, T123A181 and T123V181 expression plasmids were cocultured with RAW264.7 cells that expressed CXCL16 receptor-CXCR6. After coculture for 48 hours, nonadherent THP-1 cells were washed away, and the fluorescence intensity showed the cell adhesion ability of transmembrane forms of CXCL16. The results showed that significantly more THP-1 cells with green fluorescence expressing CXCL16-T123V181 protein were attached to RAW264.7 cells (Fig. 2D and 2E).

\section{Conservation and protein structure analysis of CXCL16}

The 123 and 181 amino acids of the CXCL16 protein are not highly conserved in 11 mammalian species and possess a high probability of protrusion. Both amino acids 123 and 181 may be located in nonconserved regions (Fig. 3A). Polymorphism Phenotyping v2 (Polyphen2, http://genetics.bwh.harvard.edu/pph2/) was employed to predict the effect of the two mutations. The results showed that the effect of the two mutations on the protein structure was BEGIEN, which meant that the two amino acid mutations would not have a destructive effect on the protein structure. Molecular modeling analysis further indicated that I123T was located on the surface of CXCL16, which might have only a slight effect on the structure of the native protein. A181V is located in the interior of the protein, which had a strong effect on the structure of the CXCL16 protein (Fig. 3B). The results of structure comparison also showed that the average root mean squared deviation (RMSD) of I123T was only 1.62, while the average RMSD of the mutation region of A181V was 1.95, which meant that the structural change of the mutation region of $A 181 \mathrm{~V}$ was greater. The structural diagram showed that the active center of the CXCL16 protein might have been changed. The $V$ ${ }^{\circledR}$ A substitution was more hydrophobic, which might reduce the size of the active center of the CXCL16 protein (Fig. 3B).

\section{Discussion}

CXCL16 is a microbially regulated chemokine that modulates the function of natural killer T cells in the lungs and colon to modulate the inflammation of these tissues (40-41). CXCL16 and its receptor CXCR6 were recently observed to be related to various inflammatory diseases, such as glomerulonephritis (42), pulmonary diseases (43), atherosclerosis (44), coronary artery disease (45), rheumatoid arthritis (46) and many inflammation-related cancers (47-49). At present, there are only four studies investigating the clinical associations between CXCL16 polymorphisms and atherosclerosis $(32,50)$, coronary heart disease (39) and multiple sclerosis (51). CXCL16 consists of four domains, and the 123rd and 181st amino acids are located in glycosylated mucin-like domains. The chemotaxis function of CXCL16 is primarily mediated by the chemokine domain. The chemokine domain can bind to CXCR6, but the glycosylated mucin-like domain can affect the conformation of the chemotaxis domain (52). To date, there is little information regarding the clinical relevance of CXCL16 polymorphisms in trauma patients with sepsis and MODS. 
Case-control studies are commonly utilized association studies employed to identify the genetic basis of diseases. One of the main limitations of this approach is inappropriate case-control matching, such as the use of healthy blood donors as a control group, which leads to population stratification. Therefore, we only chose trauma patients and followed them prospectively to determine whether those with genetic variation had different risks of posttraumatic sepsis and MODS. In this research, we investigated the potential clinical relevance of two missense SNPs of CXCL16, namely, I123T (rs1050998) and A181V (rs2277680). Our results indicated that among the four haplotypes of I123T and A181V, T123V181 was associated with an increased risk for sepsis morbidity rate and a higher MOD score in the Chongqing population. This clinical relevance of T123V181 was further confirmed in another two independent cohorts, the Zhejiang and Guizhou cohorts. We further elucidated the influence of the four haplotypes on the adhesion and chemotaxis ability of immunocytes. In vitro chemotactic experiments showed that the CXCL16-T123V181 protein enhanced the chemotaxis of immunocytes. The adhesion ability of THP-1 cells expressing T123V181 to immunocytes was also stronger than that of the other three haplotypes. Conservation and protein structure analysis of CXCL16 showed that the average RMSD of the mutation region of $A 181 \mathrm{~V}$ was 1.95 , which meant that the structural change of the mutation region of $\mathrm{A} 181 \mathrm{~V}$ was greater. The morbidity of posttraumatic sepsis and the MODS score in T123V181 carriers were higher than those in 1123A181 carriers, which might be observed because of the structural change caused by CXCL16-T123V181. The structural diagram showed that the active center of the CXCL16 protein might have changed, and the $\mathrm{V} \circledast \mathrm{C}$ substitution was more hydrophobic, which might reduce the size of the active center of the CXCL16 protein. Notably, T123 and V181 changed the structure of the CXCL16 protein active center, which led to changes in protein function and changes in adhesion and chemotaxis of CXCL16-expressing immunocytes and, finally, led to changes in posttraumatic sepsis morbidity and the MODS score.

It must be noted that our research has a number of limitations. The sample size of Guizhou and Zhejiang populations is relatively small compared with Chongqing populations. Second, it is difficult to obtain additional blood samples to detect the serum level of CXCL16 in patients with sepsis. Third, only patients of the Han nationality was recruited in this study. The Han nationality is the most populous ethnic group in China, and a biologically relevant phenotype and an ethnically consistent population might maximize the possibility of finding meaningful genetic associations. Although the number of patients from Zhejiang and Guizhou populations is relatively small to test the impact of SNPs on immunocyte function and clinical trajectory, the correlation between the 1123A181 haplotype and sepsis in the Chongqing population was verified in the Zhejiang and Guizhou populations, and these findings can be extrapolated to other populations. Effective targeted therapy for sepsis requires an understanding of the heterogeneity of the individual host response. A precise approach using biomarkers enables the identification of subgroups of patients with different host responses and specific individuals who are likely to benefit from personalized therapy. Our genetic approach may employ genomic information to classify individuals into subpopulations that differ in their susceptibility to sepsis, in the pathophysiology of sepsis, and in their response to a specific treatment according. Therefore, our results defined the functional significance of the I123A181 haplotype of $\mathrm{CXCl} 16$ for the first time and demonstrated that it might be employed as a 
biomarker for sepsis and MODS in severe trauma patients. Our genetic methods can be employed to classify individuals and their susceptibility to sepsis and MODS in different populations according to their specific genetic information.

\section{Conclusions}

We investigated the clinical relevance of two missense SNPs, I123T and A181V, in CXCL16. Notably, T123V181 haplotypes were observed to be associated with an increased risk for sepsis morbidity rate and higher MOD score in three independent cohorts. In vitro chemotactic and adhesion experiments showed that T123V181-CXCL16 enhanced the chemotaxis and adhesion ability of immunocytes. Conservation and protein structure analysis of CXCL16 showed that T123 and V181 changed the structure of the CXCL16 protein active center, possibly leading to changes in protein function and changes in the adhesion and chemotaxis of CXCL16-expressing immunocytes. Our findings provide insight into CXCL16 T123V181 haplotypes as a novel biomarker for improving the early identification of high risk for traumatic sepsis or MODS.

\section{Declarations}

\section{Ethics approval and consent to participate}

The study protocol was approved by the Ethics and Protocol Review Committee of the Army Medical University (No. TMMU2012009). Informed consent was obtained from the patients or their next of kin.

\section{Consent for publication}

Not applicable.

\section{Availability of data and material}

The datasets used for analysis during the current study are available from the corresponding author on reasonable request.

\section{Competing interests}

All authors declare that they have no competing financial interests.

\section{Funding}

This study was supported by the National Natural Science Foundation of China (81571892 and 81660317), the Projects of the State Key Laboratory of Trauma, Burns and Combined Injury (SKLYQ201901 and SKLKF201802), the Chongqing Science and Technology Talents, Chongqing Special Project for Academicians (cstc2020yszx-jcyj0008), the Clinical Research Projects of the Army Medical 
University (2018XLC3057) and the Training Plan for Innovation Ability on the Frontiers of Military Medical Research (2019CXJSB014).

\section{Authors' contributions}

$\mathrm{J}-\mathrm{HS}$ and $\mathrm{H}-\mathrm{CZ}$ were the main researchers in this study. D-L, L-C, Q-W, L-BG and D-LW were involved in the collection of blood samples and clinical data. J-W, J-D, H-H and A-QZ performed the technical work. J-D, $J-X J, L-Z$ planned the study and L-Z wrote the manuscript. All authors read and approved the final manuscript.

\section{Acknowledgments}

We are grateful to Dr. Dong-po Jiang, Dr. Lianyang Zhang, Army Medical University, and Dr. Dingyuan Du, Chongqing Emergency Medical Center, for the collection of the blood samples.

\section{References}

1. Dellinger RP, Levy MM, Rhodes A, et al: Surviving Sepsis Campaign: International Guidelines for Management of Severe Sepsis and Septic Shock: 2012. Crit Care Med 2013; 41(2):580-637

2. Seymour CW, Gesten F, Prescott HC, et al: Time to Treatment and Mortality during Mandated Emergency Care for Sepsis. N Engl J Med 2017; 376 (23):2235-2244

3. Prescott HC, Angus DC: Enhancing Recovery From Sepsis: A Review. JAMA 2018; 319(1):62-75

4. Fleischmann C, Scherag A, Adhikari NK, et al: Assessment of Global Incidence and Mortality of Hospital-treated Sepsis. Current Estimates and Limitations. Am J Respir Crit Care Med 2016; 193(3):259-72

5. Sorensen TI, Nielsen GG, Andersen PK, et al: Genetic and enviromental influences on premature death and in adult adoptees. N Engl J Med 1988; 318(12):727-732

6. Gu W, Jiang JX: Genetic Polymorphisms and Posttraumatic Complications. Comp Funct Genomics 2010;814086

7. Stuber F, Petersen M, Bokelmann F, et al: A genomic polymorphism within the tumor necrosis factor locus influences plasma tumor necrosis factor-alpha concentrations and outcome of patients with severe sepsis. Crit Care Med 1996; 24(3):381-384

8. Zeng L, Gu W, Zhang AQ, et al: A functional variant of lipopolysaccharide binding protein predisposes to sepsis and organ dysfunction in patients with major trauma. Ann Surg 2012; 255(1):147-157.

9. Zeng L, Zhang AQ, Gu W, et al: Identification of Haplotype tag SNPs Within the Whole Myeloid Differentiation-2 Gene and Their Clinical Relevance in Patients with Major Trauma. Shock 2012; $37(4): 366-372$

10. Gu W, Zeng L, Zhou J, et al: Clinical relevance of 13 cytokine gene polymorphisms in Chinese major trauma patients. Intens Care Med 2010; 36(7):1261-1265 
11. Zeng L, Gu W, Chen K, et al: Clinical relevance of the interleukin 10 promoter polymorphisms in Chinese Han patients with major trauma: genetic association studies. Crit Care 2009; 13(6):R188

12. Zeng L, Zhang AQ, Gu W, et al: Clinical relevance of single nucleotide polymorphisms of the high mobility group box 1 protein gene in patients with major trauma in Southwest China. Surgery 2011; 151(3):427-436

13. McDonald B, Urrutia R, Yipp BG, et al. Intravascular neutrophil extracellular traps capture bacteria from the bloodstream during sepsis. Cell Host Microbe 2012; 12(3):324-333

14. Allam R, Kumar SV, Darisipudi MN, et al. Extracellular histones in tissue injury and inflammation. $J$ Mol Med (Berl) 2014; 92(5):465-472

15. Paudel S, Baral P, Ghimire L, et al: CXCL1 regulates neutrophil homeostasis in pneumoniaderived sepsis caused by Streptococcus pneumoniae serotype 3. Blood 2019; 133(12):1335-1345

16. Satake $\mathrm{S}$, Hirai $\mathrm{H}$, Hayashi $\mathrm{Y}$, et al: $\mathrm{C} / \mathrm{EBPb}$ is involved in the amplification of early granulocyte precursors during candidemiainduced "emergency" granulopoiesis. J Immuno/ 2012; 189(9):45464555

17. Zhang H, Nguyen-Jackson H, Panopoulos AD, et al: STAT3 controls myeloid progenitor growth during emergency granulopoiesis. Blood 2010; 116(14):2462-2471

18. Wang Z , Rui T, Yang M, et al: Alveolar macrophages from septic mice promote polymorphonuclear leukocyte transendothelial migration via an endothelial cell Src kinase/NADPH oxidase pathway. $J$ Immuno/ 2008; 181(12):8735-44

19. Wuttge DM, Zhou X, Sheikine Y, et al: CXCL16/SR-PSOX is an interferon-regulated chemokine and scavenger receptor expressed in atherosclerotic lesions. Arterioscler Thromb Vasc Biol 2004; 24:750755

20. Shimaoka T, Kume N, Minami M, et al: Molecular cloning of a novel scavenger receptor for oxidized low density lipoprotein, SR-PSOX, on macrophages. J Biol Chem 2000; 275:40663- 40666

21. Wilbanks A, Zondlo SC, Murphy K, et al: Expression cloning of the STRL33/BONZO/ TYMSTR ligand reveals elements of CC, CXC, and CX3C chemokines. J Immunol 2001; 166:5145-5154

22. Matloubian $M$, David $A$, Engel $S$, et al: $A$ transmembraneCXC chemokine is a ligand for HIVcoreceptor Bonzo. Nat Immunol 2000; 1:298 -304

23. Kim MJ, Sun HJ, Song YS, et al: CXCL16 positively correlated with M2-macrophage infiltration, enhanced angiogenesis, and poor prognosis in thyroid cancer. Sci Rep 2019; 9(1):13288

24. Yamauchi R, Tanaka M, Kume N, et al: Upregulation of SR-PSOX/CXCL16 and recruitment of CD8+ T cells in cardiac valves during inflammatory valvular heart disease. Arterioscler Thromb Vasc Biol 2004; 24(2):282-7.

25. Carossino M, Dini P, Kalbfleisch TS, et al: Equine arteritis virus long-term persistence is orchestrated by CD8+ T lymphocyte transcription factors, inhibitory receptors, and the CXCL16/CXCR6 axis. Plos Pathog 2019; 15(7):e1007950 
26. Lepennetier G, Hracsko Z, Unger M, et al: Cytokine and immune cell profiling in the cerebrospinal fluid of patients with neuro-inflammatory diseases. J Neuroinflammation 2019; 16(1):219

27. Tohyama M, Sayama K, Komatsuzawa $\mathrm{H}$, et al: CXCL16 is a novel mediator of the innate immunity of epidermal keratinocytes. Int Immunol 2007; 19(9):1095-1102

28. Borst $\mathrm{O}$, Schaub M, Walker B, et al: CXCL16 is a novel diagnostic marker and predictor of mortality in inflammatory cardiomyopathy and heart failure. Int J Cardiol 2014; 176(3):896-903

29. Fahy OL, Townley SL, McColl SR. CXCL16 regulates cell-mediated immunity to Salmonella enterica serovar Enteritidis via promotion of gamma interferon production. Infect Immun 2006; 74(12):68856894

30. Shimaoka T, Nakayama T, Kume N, et al: SR-PSOX/CXC chemokine ligand 16 mediates bacterial phagocytosis by APCs through its chemokine domain. $J$ Immunol 2003; 171(4):1647-51

31. Xu S, Cheng J, Cai MY, et al: The Impact of tagSNPs in CXCL16 Gene on the Risk of Myocardial Infarction in a Chinese Han Population. Dis Markers 2017; 2017:9463272

32. Zivković M, Djurić T, Stojković L, et al: CXCL16 haplotypes in patients with human carotid atherosclerosis preliminary results. $J$ Atheroscler Thromb 2015; 22(1):10-20

33. Singer M, Deutschman CS, Seymour CW, et al: The Third International Consensus definitions for sepsis and septic shock (Sepsis-3). JAMA 2016; 315:801-810

34. Marshall JC, Cook DJ, Christou NV, et al: Multiple organ dysfunction score: a reliable descriptor of a complex clinical outcome. Crit Care Med 1995; 23:1638-1652

35. Lu H, Wen D, Sun J, et al: Enhancer Polymorphism rs10865710 Associated With Traumatic Sepsis Is a Regulator of PPARG Gene Expression. Crit Care 2019; 23(1):430

36. Li C, Zhao J, Sun L, et al: RANKL Downregulates Cell Surface CXCR6 Expression Through JAK2/STAT3 Signaling Pathway During Osteoclastogenesis. Biochem Biophys Res Commun 2012; 429(3-4):156-162

37. Gan L, Hu C, Deng Z, et al: Rs1982809 is a functional biomarker for the prognosis of severe posttraumatic sepsis and MODs. Exp Biol Med (Maywood) 2019; 244(16):1438-1445

38. Daly MJ, Rioux JD, Schaffner SF, et al: High-resolution haplotype structure in the human genome. Nat Genet 2001;29(2):229-232

39. Petit SJ, Wise EL, Chambers JC, et al: The CXCL16 A181V mutation selectively inhibits monocyte adhesion to CXCR6 but is not associated with human coronary heart disease. Arterioscler Thromb Vasc Biol 2011;31(4):914-20.

40. Abel S, Hundhausen C, Mentlein R, et al: "The transmembrane CXC-chemokine ligand 16 is induced by IFN-gamma and TNF-alpha and shed by the activity of the disintegrin-like metalloproteinase ADAM10". J Immunol 172 (10): 6362-72

41. Olszak T, An D, Zeissig S, et al: Microbial exposure during early life has persistent effects on natural killer T cell function. Science, 336 (6080):489-493 
42. Garcia GE, Truong LD, Li P, et al: Inhibition of CXCL16 attenuates inflammatory and progressive phases of anti-glomerular basement membrane antibody-associated glomerulonephritis. Am J Pathol 2007; 170(5):1485-96

43. Zuo S, Zhu Z, Liu Y, et al: CXCL16 Induces the Progression of Pulmonary Fibrosis through Promoting the Phosphorylation of STAT3. Can Respir J 2019; 2019:2697376

44. Aslanian AM, Charo IF: Targeted disruption of the scavenger receptor and chemokine CXCL16 accelerates atherosclerosis. Circulation 2006; 114(6):583-90

45. Zhou F, Wang J, Wang K, et al: Serum CXCL16 as a Novel Biomarker of Coronary Artery Disease in Type 2 Diabetes Mellitus: a Pilot Study. Ann Clin Lab Sci 2016; 46(2):184-9

46. van Lieshout AW, van der Voort R, Toonen LW, et al: Regulation of CXCL16 expression and secretion by myeloid cells is not altered in rheumatoid arthritis. Ann Rheum Dis 2009; 68(6):1036-1043

47. Singh R, Kapur N, Mir H, et al: CXCR6-CXCL16 axis promotes prostate cancer by mediating cytoskeleton rearrangement via Ezrin activation and av $\beta 3$ integrin clustering. Oncotarget 2016; 7(6):7343-7353

48. Chung B, Esmaeili AA, Gopalakrishna-Pillai S, et al: Human brain metastatic stroma attracts breast cancer cells via chemokines CXCL16 and CXCL12. NPJ Breast Cancer 2017; 3:6

49. Kee JY, Ito A, Hojo S, et al: CXCL16 suppresses liver metastasis of colorectal cancer by promoting TNF-a-induced apoptosis by tumor-associated macrophages. BMC Cancer 2014; 14:949

50. Wang KD, Liu ZZ, Wang RM, et al: Chemokine CXC Ligand 16 serum concentration but not A181V genotype is associated with atherosclerotic stroke. Clinica Chimica Acta 2010; 411(19-20):14471451

51. Stojković L, Stanković A, Djurić T, et al: The gender-specific association of CXCL16 A181V gene polymorphism with susceptibility to multiple sclerosis, and its effects on PBMC mRNA and plasma soluble CXCL16 levels: preliminary findings. J Neuro/ 2014; 261(8):1544-1551

52. Petit SJ, Chayen NE, Pease JE: Site-directed mutagenesis of the chemokine receptor CXCR6 suggests a novel paradigm for interactions with the ligand CXCL16. Eur J Immunol 2008; 38(8):2337-2350

\section{Tables}

Table 1. Overall clinical characteristics of patients with major trauma 


\begin{tabular}{|c|c|c|c|}
\hline & \multirow{2}{*}{$\begin{array}{l}\text { Screening cohort } \\
\text { Chongqing }(\mathrm{N}=920)\end{array}$} & \multicolumn{2}{|l|}{ Validation cohorts } \\
\hline & & Zhejiang(N=350) & Guizhou(N=350) \\
\hline Age (yrs) & $43.5 \pm 15.1(18-65)$ & $42.5 \pm 12.0(19-62)$ & $37.6 \pm 12.5(18-65)$ \\
\hline Male/female, $n$ & $681 / 239$ & $268 / 82$ & $273 / 77$ \\
\hline \multicolumn{4}{|l|}{ Injured body regions, n (\%) } \\
\hline Head, $n$ & $475(51.6)$ & $224(64.0)$ & $207(59.1)$ \\
\hline Thorax, $\mathrm{n}$ & $536(58.2)$ & $216(61.7)$ & $198(56.6)$ \\
\hline Abdomen, $\mathrm{n}$ & $381(41.4)$ & $129(36.9)$ & $116(33.1)$ \\
\hline Extremities, $\mathrm{n}$ & $416(45.2)$ & $198(56.6)$ & $187(53.4)$ \\
\hline \multicolumn{4}{|l|}{ Number of regions injured, n (\%) } \\
\hline One, $n$ & $461(50.1)$ & $151(43.1)$ & $146(41.7)$ \\
\hline Two, $n$ & $292(31.7)$ & $132(37.7)$ & $129(36.9)$ \\
\hline Three or above, $n$ & $167(18.2)$ & $67(19.1)$ & $75(21.4)$ \\
\hline ISS & $23.4 \pm 9.8$ & $22.4 \pm 8.1$ & $21.5 \pm 9.1$ \\
\hline $316,<25, \mathrm{n}(\%)$ & $567(61.6)$ & $201(57.4)$ & $221(63.1)$ \\
\hline${ }^{3} 25, \mathrm{n}(\%)$ & $353(38.7)$ & $149(42.6)$ & $129(36.9)$ \\
\hline \multicolumn{4}{|l|}{ Organ dysfunction, n (\%) } \\
\hline \multicolumn{4}{|l|}{ None, $\mathrm{n}$} \\
\hline One, $\mathrm{n}$ & $281(32.2)$ & $112(32.9)$ & $125(34.1)$ \\
\hline Two, $n$ & $121(14.5)$ & $56(16.5)$ & $51(13.9)$ \\
\hline Three or above, $n$ & $41(4.9)$ & $23(6.8)$ & $35(9.5)$ \\
\hline Sepsis, n (\%) & 347 (37.7) & $118(33.7)$ & 132(37.7) \\
\hline \multicolumn{4}{|l|}{ Source of infection, \% } \\
\hline Respiratory tract infection & 42.3 & 40.5 & 42.8 \\
\hline Primary bloodstream infection & 22.1 & 22.9 & 20.3 \\
\hline Urinary tract infection & 15.0 & 12.5 & 13.1 \\
\hline Catheter associated infection & 10.6 & 8.2 & 9.5 \\
\hline Wound infection & 7.3 & 8.8 & 10.2 \\
\hline Others* & 2.7 & 7.1 & 4.1 \\
\hline
\end{tabular}


Table 2. Distribution of haplotypes of CXCL16 I123T and A181V among trauma patients in three cohorts

\begin{tabular}{|lllll|}
\hline Study Cohort & I123A181 $(\mathrm{n}, \%)$ & I123V181 $(\mathrm{n}, \%)$ & $\begin{array}{l}\text { T123A181 } \\
(\mathrm{n}, \%)\end{array}$ & $\begin{array}{l}\text { T123V181 } \\
(\mathrm{n}, \%)\end{array}$ \\
\hline Chongqing & $548(59.57)$ & $3(0.33)$ & $1(0.11)$ & $368(40.0)$ \\
\hline Zhejiang & $182(52.0)$ & $0(0)$ & $1(0.29)$ & $167(47.71)$ \\
\hline Yunnan & $176(50.29)$ & $2(0.57)$ & $0(0)$ & $172(49.14)$ \\
\hline
\end{tabular}

Table 3. Haplotype effects of the I123T and A181V polymorphisms on the incidence of sepsis among trauma patients in three cohorts 


\begin{tabular}{|c|c|c|c|c|c|c|c|}
\hline & Haplotypes & $\mathbf{N}$ & Age(yr) & $\operatorname{Sex}(M / F)$ & ISS & Sepsis, n/[ & $\begin{array}{l}\text { MOD } \\
\text { score }\end{array}$ \\
\hline \multirow[t]{5}{*}{ Chongqing } & I123A181 & 548 & $44.2 \pm 12.9$ & $379 / 136$ & $22.6 \pm 9.3$ & $\begin{array}{l}181(33.0 \%) \\
\text { a1 }\end{array}$ & \\
\hline & & & & & & & 5 \\
\hline & I123V181 & 3 & $43.5 \pm 13.7$ & $2 / 1$ & $21.5 \pm 5.7$ & $0(\%)$ & $6.6 \pm 1.3$ \\
\hline & T123A181 & 1 & 48.0 & $1 / 0$ & 20.0 & $0(\%)$ & 7 \\
\hline & T123V181 & 368 & $43.7 \pm 13.3$ & 299/102 & $21.6 \pm 9.4$ & $166(45.1 \%)$ & $7.8 \pm 2.7$ \\
\hline \multirow[t]{4}{*}{ Zhejiang } & I123A181 & 182 & $42.6 \pm 11.8$ & $138 / 44$ & $22.3 \pm 7.1$ & $49(26.9 \%)^{\mathrm{a} 2}$ & $6.7 \pm 3.0^{b 2}$ \\
\hline & I123V181 & 0 & - & - & - & - & - \\
\hline & T123A181 & 1 & 46 & $1 / 0$ & 22.0 & $1(100 \%)$ & 7.0 \\
\hline & T123V181 & 167 & $43.5 \pm 14.7$ & $129 / 38$ & $19.7 \pm 7.5$ & $68(40.7 \%)$ & $8.1 \pm 2.9$ \\
\hline \multirow[t]{4}{*}{ Guizhou } & I123A181 & 176 & $37.4 \pm 12.5$ & $141 / 42$ & $22.8 \pm 9.2$ & $45(25.6 \%)^{a 3}$ & $6.9 \pm 2.5^{b 3}$ \\
\hline & I123V181 & 2 & $38.5 \pm 12.0$ & $2 / 0$ & $21.5 \pm 3.0$ & $0(\%)$ & $7.5 \pm 2.6$ \\
\hline & T123A181 & 0 & - & - & - & - & - \\
\hline & T123V181 & 172 & $39.8 \pm 11.9$ & $130 / 35$ & $22.6 \pm 8.9$ & $87(50.6 \%)$ & $8.5 \pm 2.9$ \\
\hline
\end{tabular}

a:dominant effect (variant homozygotes theterozygotes vs. wild homozygotes) as analyzed by ANCOVA, ${ }^{a 1} \mathrm{P}=0.001,{ }^{a} \mathrm{P}=0.004,{ }^{\mathrm{a} 3} \mathrm{P}=0.012$. b: recessive effect (variant homozygotes vs. heterozygotes + wild homozygotes) as analyzed by ANCOVA, ${ }^{\mathrm{b} 1} \mathrm{P}=0.0016,{ }^{\mathrm{b} 2} \mathrm{P}=0.002,{ }^{\mathrm{b} 3} \mathrm{P}=0.001$.

\section{Figures}


A

I123T A181V

A

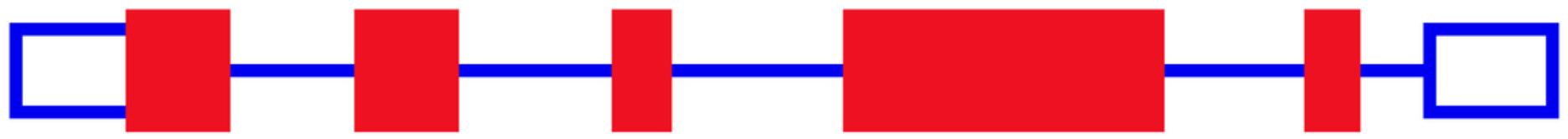

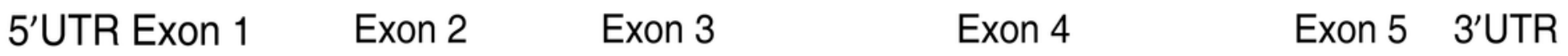

B
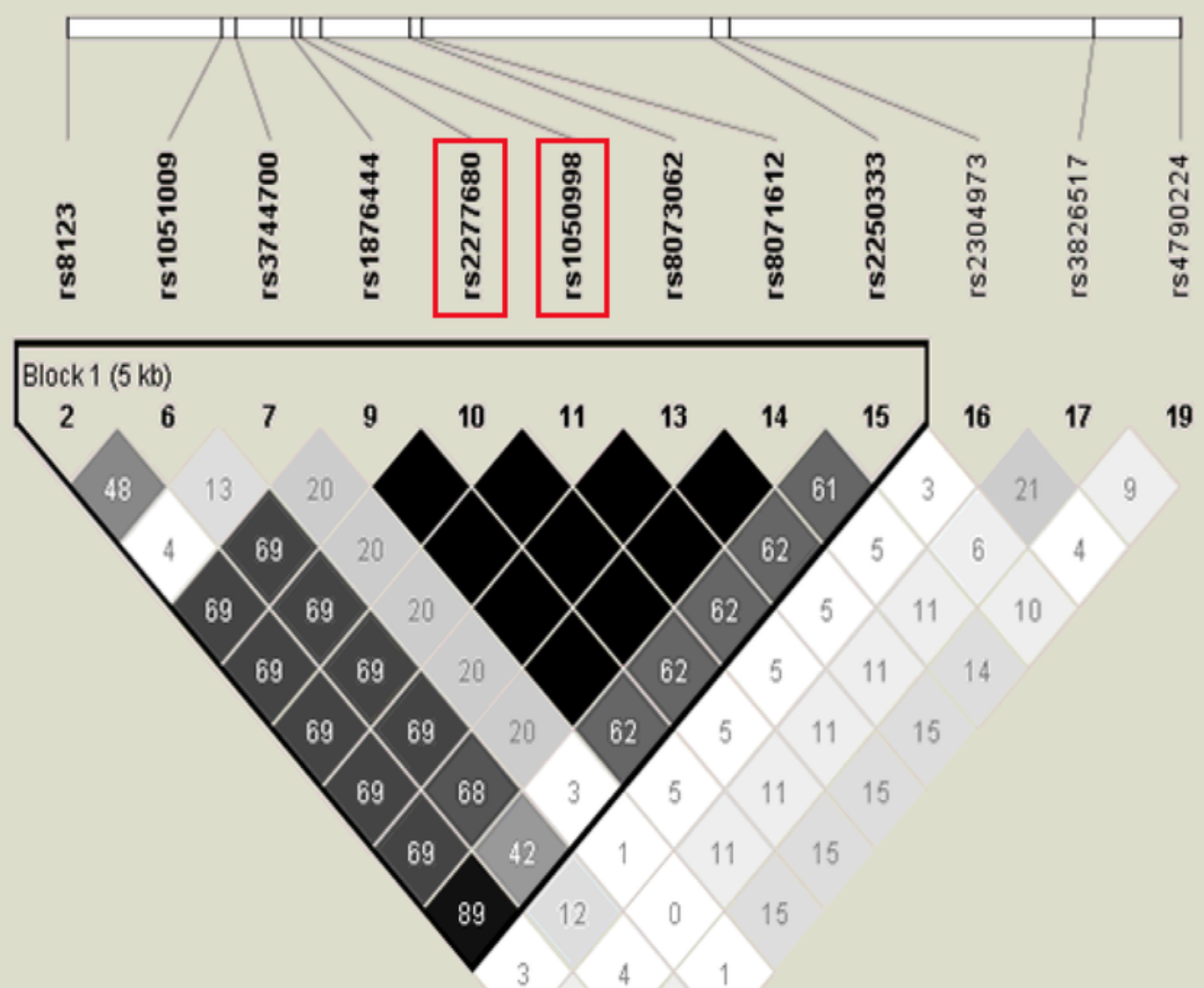

\section{Figure 1}

Overview of tagging single nucleotide polymorphisms (tag SNPs) in the CXCL16 gene. (A) Location of SNPs in the CXCL16 gene with a minor allele frequency $\geq 5 \%$. A linkage disequilibrium (LD) plot of these SNPs is displayed by a color scheme. Black represents very high LD $(r 2=1.0)$, and white indicates the absence of correlation $(\mathrm{r} 2=0$ ) between two SNPs. The $\mathrm{r} 2$ between I123T (rs1050998) and A181V 
(rs2277680) is 1.0. (B) CXCL16 gene organization and the location of two missense mutations, I123T and A181V, on chromosome 17.

A

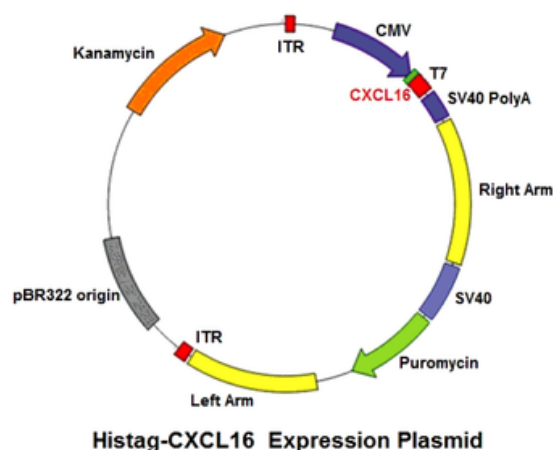

Histag-CXCL16 Expression Plasmid

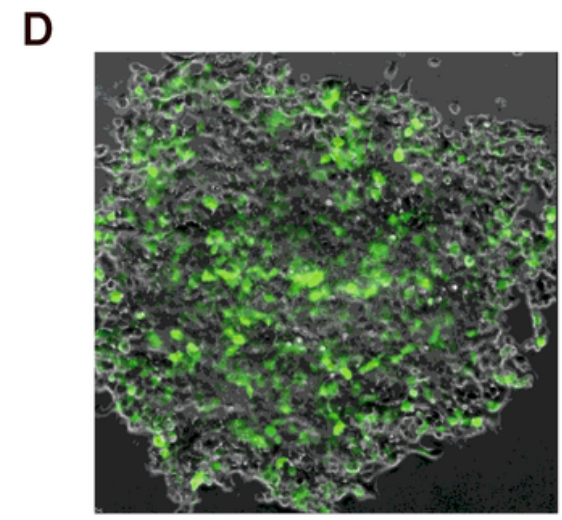

|123A181
B
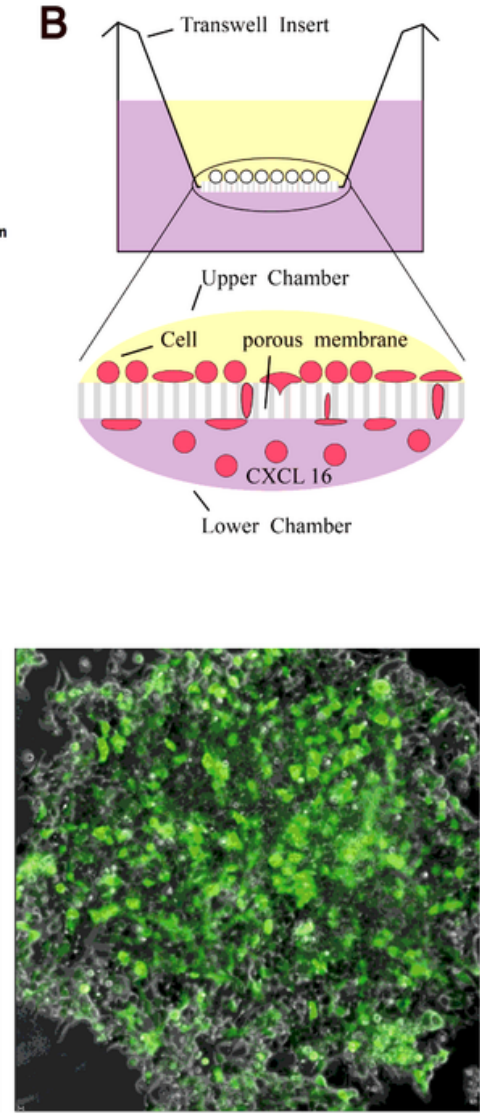

T123V181
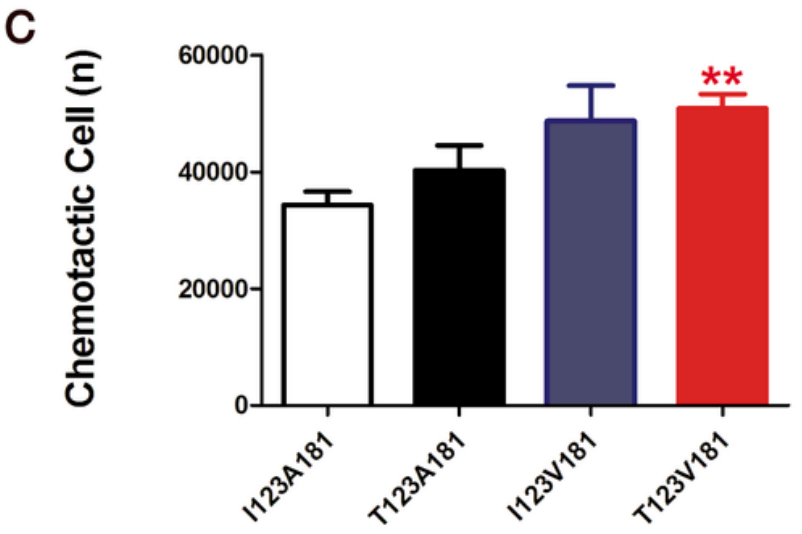

E

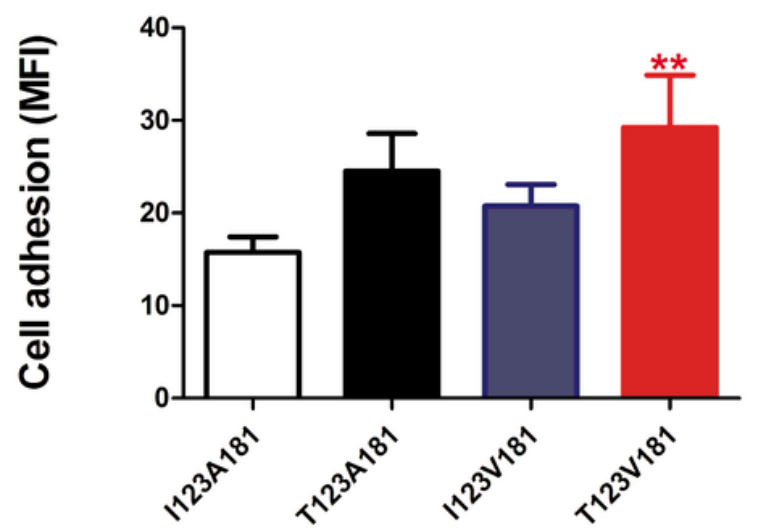

Figure 2

T123V181 haplotype enhances the chemotaxis and adhesion of inflammatory cells. (A) Map of the CXCL16 expression plasmid. (B) Schematic diagram of the chemotactic assay. The percentage of THP-1 cells recruited to the lower chamber was calculated to represent chemotaxis. (C) The chemotaxis of the CXCL16-T123V181 protein was significantly higher than that of the other three proteins ( $\left.{ }^{\star \star P}<<0.01\right)$. (D) The percentage of THP-1 cells with green fluorescence expressing transmembrane forms of CXCL16T123V181 protein attached to RAW264.7 cells was calculated to represent the adhesion ability. (F) The adhesion ability of cells expressing CXCL16-T123V181 protein was significantly higher than that of the other three proteins $(* * \mathrm{P}<0.01)$. 
A

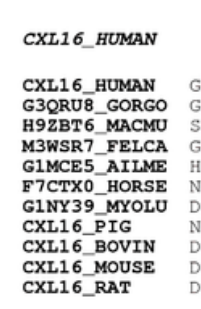

$\stackrel{\alpha 2}{2}$

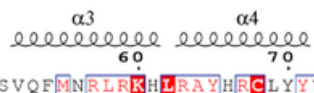
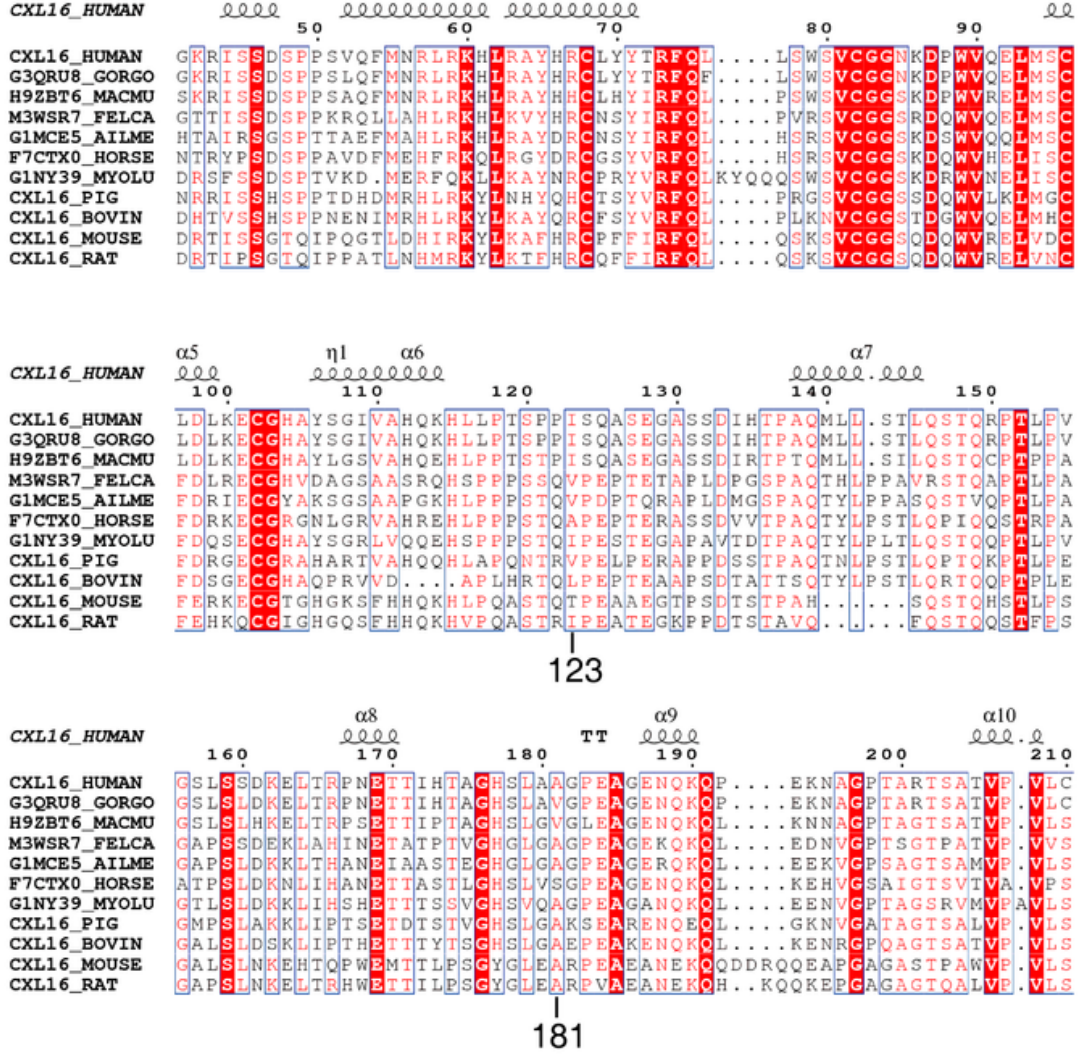

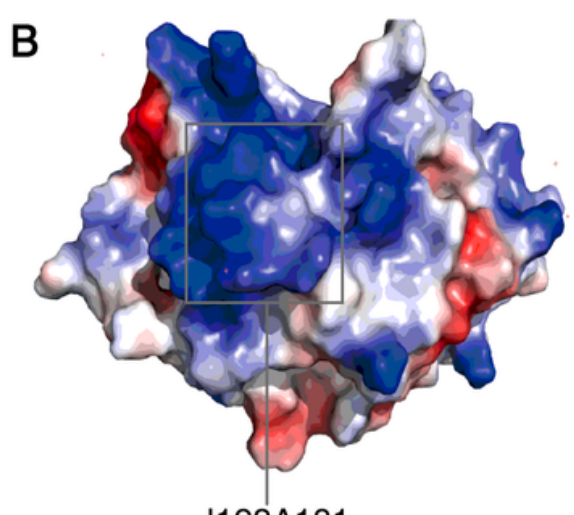

I123A181

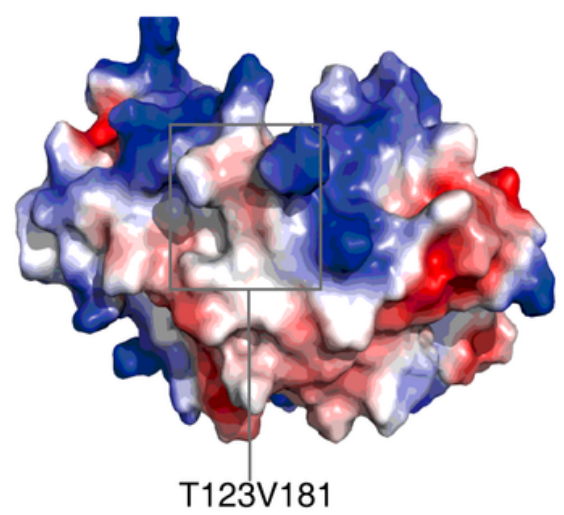

\section{Figure 3}

Conservative analysis and molecular modeling of I123T and A181V. (A) Conservative analysis analysis shows that the two missense mutations are both located in the nonconserved region of the CXCL16 gene. (B) The structural diagram shows that the active center of the CXCL16 protein was changed by $\mathrm{V} \rightarrow \mathrm{A}$ substitution, which might make the active center of the CXCL16 protein smaller. 